\title{
Oil/Gas Pipeline Leak Inspection and Repair in Underwater Poor Visibility Conditions: Challenges and Perspectives
}

\section{Agbakwuru Jasper}

Offshore Engineering, Faculty of Science and Technology, University of Stavanger, Stavanger, Norway.

Email: jasper.agbakwuru@uis.no

Received February 29 ${ }^{\text {th }}$, 2012; revised March 22 ${ }^{\text {nd }}, 2012$; accepted April $15^{\text {th }}$, 2012

\begin{abstract}
Mechanical pressure clamps are examples of innovative tools commonly used in the oil and gas industry for arresting leaks from damaged oil and gas pipelines. However, if leaks result from pipeline rupture, clamps are not usually recommended. It is therefore obvious that inspection of the leaking pipeline is very crucial in deciding the strategy for repair. For subsea pipelines where underwater poor visibility is pronounced, this important aspect of the pipeline repair process becomes difficult to implement. The result is a repair-leak-repair cycle. This challenge is commonly found in repairs of old pipelines in unclear water conditions. Old pipelines and their vulnerability to fractures that often lead to ruptures are discussed. In this paper, the challenges and technologies available for visualisation and examination in such unclear water conditions are discussed. There appears to be a gap in the existing pipeline integrity management system with respect to inspection and repair of pipelines in unclear water conditions. This gap needs to be filled in order to minimise spills and pollution. For pipelines installed in unclear water condition, a perspective is suggested to extend the capability of existing remotely operated vehicles to employ the use of clear laminar water system or a related technique to provide integrity engineers and operators with close visual assess to inspect leaking pipelines and effect adequate repairs. This paper suggests that the use of optical eye as the main tool for examination remains valuable in managing the challenges in underwater pipeline repairs in unclear water condition.
\end{abstract}

Keywords: Pipeline; Leaks; Detection; Spill; Close-Visual Inspection; Pipeline Repair; Pipeline Inspection

\section{Introduction}

The pipeline integrity engineer takes responsible care of the pipeline system by applying an adequate Pipeline Integrity Management System (PIMS). The pipeline integrity management system encompasses all efforts to ensure the pipeline is secured and safe to operate. This implies adequate inspection and maintenance, ensuring fit-for-service, operational control and monitoring, adequate repair systems in place should the need arise, trained personnel, regular audits and risk assessments etc. and more importantly, a management system to harness these activities. These efforts create an opportunity for the pipeline operator to have full control over the pipeline life and functionality.

There is today, evidence of repair-leak-repair cycle involving underwater pipelines in poor visibility conditions [1]. This is particularly common in pipelines often classified as "old" pipelines. This is against the object of PIMS and represents a gap. The poor underwater visibility is often due to inorganic and particulates of organic substances swept into the open water or sea by tidal actions, rain water, wind, industrial processes etc. This problem creates room for oil spills with its attendant social, economic and environmental damages.

It is also noted that in some developing countries, there is an increasing trend for inspection of damages on pipelines by joint investigation teams to determine whether a spill is caused intentionally or not. Unfortunately, the joint inspection exercise is a prerequisite requirement for pipeline repairs in some regions of the world [2]. It is therefore desirable that agreement between the stakeholders in the joint investigation team be resolved as quickly as possible using a suitable close visual inspection or related technique. Quick resolution of causes of damages for submerged pipeline causing spills in unclear/muddy water may be difficult with the present industry tools.

This paper intends to explore the reasons for the inspection and repair difficulties and proposes an approach towards development of systems that could provide effective solution to oil/gas/liquid pipeline inspection and 
adequate repair strategy for leaking pipelines in underwater poor visibility conditions.

\section{Aged Pipelines and Their Vulnerability}

Line-pipes made prior to the early 1970s are often considered as aged or old pipelines. Their metallurgical constituents and external corrosion coating effectiveness are of lower standard compared to line-pipes of today. While today's modern line-pipes can have Charpy toughness values $\mathrm{Cv}$, up to $300 \mathrm{~J}$, the aged pipelines have toughness values within $\mathrm{Cv}, 2 / 3,40 \mathrm{~J}[3,4]$. (Note that $\mathrm{J}$ is energy release rate at crack tips of non-elastic material such as pipeline. The $2 / 3$ implies $75 \%$ of the standard size of test specimen, $10 \times 10 \times 5.5 \mathrm{~mm}$ ). Toughness is the ability of the steel to withstand crack or crack propagation. Furthermore, poor external coating means that cathodic and environmental processes on the surfaces of structures could lead to hydrogenation problems within the steel matrix, further reducing the toughness values. Hydrogenation is a process of diffusion and positioning of hydrogen atom within the atomic structure of the parent steel, stiffening the atomic structure. Overall significance is that impacts on these pipelines would lead to large stresses and damages which have propensity to propagate. This propensity is due to lower ductility. Therefore, brittle fracture could be the failure mode for old pipelines.

Many old pipelines still exist. About 50\% of United States oil and gas pipelines are over 40 years of age [5]. In Nigeria, West Africa, many oil/gas/liquid pipelines are 30 to 50 years of age [6]. There could be different reasons the pipelines have remained in service. It could be economic, technological, political, social, security or even combinations of one or more factors. Unfortunately, many of the pipelines are in hash corroding environment. Therefore, they represent, a major engineering challenge considering the polluting impact they cause when spill occurs.

\section{Underwater Poor Visibility}

"Underwater poor visibility and unclear water" is a definition given in this paper for the description of water, be it river, ocean, sea or lake, where the ability to see through or within is impaired. The result is that close observations or inspections of items existing below the water surface become difficult and in some cases, impossible. The particulates in the water, absorb and scatter sunlight and as the light passes through them, poor visibility or unclear water results.

The geography and topographical make up of certain regions of the world most often facilitate this phenomenon. For instance, in Nigeria, West Africa the topography channel runoffs via rivers to common targets, made of loosely sediments and with the coastal areas and delta underlain by soft geologically young loosely sediments [7]. Rainfalls then sweep the whole large area of the region, coupled with regular tides that wash the coast-lines, and deposit the contents to the coastal waters. The consequence is that the littoral area, from swamps to the coast and in some instance, some miles from shore is characterized by "unclear water". Unfortunately, pipelines from oil and gas fields run through these areasswamps, deltas, littoral zones and some miles from shore towards offshore, and often, underwater inspections and repairs are required to be carried out.

\subsection{Underwater Pipeline Leak Repairs and Poor Visibility Problems}

As defined by DNV-RP-F113, [8], a pipeline repair in general requires a range of planning and investigations prior to the actual repair. This includes but is not limited to investigation of the damage, the pipe condition and consequences for the pipeline operation, planning of uncovering and seabed preparation for the repair including calculations of the pipeline response from this action etc., [8]. It is needless to say that the practice would be difficult to execute where the underwater visibility is poor. This is a pointer to the problem.

In practice, when there is leak from oil and gas pipelines, the first attempt is to reduce (or zero) the pressure and contain the leak. The next line of action is to inspect and repair, preferably, clamp the leaking section and resume production. Even when there are other subsea pipeline repair methods, mechanical pressure sealing clamps are widely used by the industry.

The mechanical pressure containing clamps are usually considered as temporary repairs, however, many subsea clamps are often not replaced and become a permanent repair subject to regular inspections [5]. Several types of the clamp include Splidco, PII Tecnomarine, Hydrotech, Furmanite etc.

For the underwater pipeline repair technique, in a simple term, overburden/soil must be cleared and the concrete/corrosion coating removed for a length of about $100 \mathrm{~cm}$ (40"). The repair clamp can then be lowered into position over the pipe and set. These operations can be done by divers. In deep water, these can be performed by Remotely Operated Vehicles in pipeline sizes to $45.7 \mathrm{~cm}$ (18”) [5].

In underwater conditions where the visibility is poor, operations as described above become difficult to perform by either ROV or diver. The inability to visually see to inspect, prepare and repair pipelines using these innovative pressure containing clamps is a challenge to the 
integrity of underwater pipelines in poor underwater conditions.

An important consideration for use of mechanical clamps is the nature of "break" on the pipeline. The mechanical clamps are designed to contain leaks, i.e., a through-hole with no propensity to propagate [5,8]. This is usually pin-holes or through-hole corrosion leaks. Ruptures, especially in old pipelines do however, have the potential to propagate, and mechanical clamps are not usually recommended. Ruptures could result from damages such as burst, sharp breaks from gouges, dents/ gouge combination, intended and unintended damages. To make this classification, visualising the break is often required. The algorithm could be in the form as shown in Figure 1.

In some developing countries, due to numerous reports of intended encroachment especially to oil pipelines, it has become a standard practice to conduct a Joint Inspection Visit (JIV) to ascertain the factor responsible for a leak/spill. This is more pronounced in joint venture production oil/gas field licences where the operating company may be required to incur the cost of damages for leak/spill due to operational reasons or avoidable structural problems. For instance, narrating the practice in Shell Nigeria, when an oil spill occurs, a joint investigation team visits the site as quickly as possible to establish the cause and volume of the spill. The team is led by Shell Nigeria, and representatives from regulatory bodies and the Ministry of Environment. In addition, the police, state government officials and impacted communities are also invited to attend the visit. In cases where the investigation shows that the spill is within Shell's control, Shell negotiates with the affected landowners [2] and they are compensated. Nigerian law does not require payment of compensation in cases of sabotage. In many cases, the community and oil field operators disagree on the cause of a spill [9]. Local communities as stakeholder

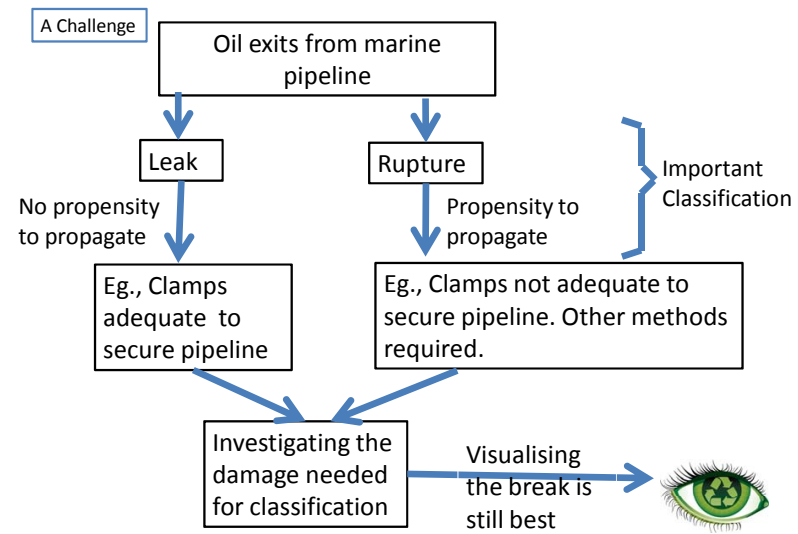

Figure 1. Criteria for underwater pipeline leak repair using mechanical clamps. and participants in Joint Inspection Team appreciate visual imaging of the scenario.

This investigation is often achieved by means of a Close-visual Inspection. Close-visual inspection is an important component of an inspection with human eyes as the main tool. It is often carried out using any of these three methods: use of human eye from close quarters, close-up still photography and close-up video recording [10]. The disagreement between stakeholders could be high in underwater poor visibility conditions. Unfortunately, the practice of joint investigation between stakeholders, landlords (communities) and oil pipeline operator are often completed before the actual pipeline repair is done. This would probably increase pollution due to intrusion processes [11].

\subsection{Underwater Pipeline Leak Inspection and Poor Visibility Issues}

Inspection techniques that can be carried out by an underwater diver or equipped Remotely Operated Vehicle in underwater poor visibility condition are discussed in this chapter. The latent challenge is tracking the leaking hole from the surface through the unclear water mass.

\section{- Hydrophone/Acoustic sensors}

These are effectively underwater microphones that "listen" for ultrasonic generated by leaking fluids under pressure. The acoustic signals generated by a leak tend to be at frequencies well above the audible range thus requiring sophisticated sensors and software to reliably determine the difference between leak generated and ambient "noise". The major problems with this method are the sounds caused by the attendant ROV and other vessels in the vicinity. However, modern data handling and spectral analysis techniques have improved the method sufficiently such that in the right conditions, the method can be highly successful [12]. These conditions cannot be met in all underwater leaks. For instance, low pressure, leakage from a large opening of liquid content pipeline would likely yield little or no tractable acoustic signal underwater.

\section{- Direct hydrocarbon leak detection}

Hydrocarbons in oil form such as crude oil can be detected using leak detectors (e.g., the Neptunes Long Range leak detector type tool), a different method would be required for gas. In some modern systems, a direct reading hydrocarbon sensor used is essentially a general hydrocarbon detector that will respond to most hydrocarbons, however, the potential for oil contamination on the sensors membrane makes it less suitable for oil detection but good for gas detection. The very high sensitivity of the sensor makes it ideal for the detection of gas seepage from the seabed [12]. 


\section{- Fluorescence}

ROV's equipped with optic systems with black light for fluorescence. Traditionally, leaks have been found using fluorescent dyes detected by 'black light (unfiltered ultra-violet) light. The major problem with this is that the dye concentration has been very high and visibility must be good. Another problem with the un-tuned "black light" is that some marine organisms fluorescence thus causing the observer to see a "fog" that masks the location of the leak [12]. Deploying submersible "tuned" fluorometers that send data up to the attendant vessel providing a real time visual display especially in clear water condition has largely solved this problem. In opaque water however, challenges still exist.

\section{Could There Be Alternatives to Human Eye in Close Visual Inspection?}

Laser Scan, Fibre Optic, Ultrasonic and Acoustic Imaging Methods:

There are visual systems today that have the capacity to capture the surfaces of objects and provide real 3-dimensional (3-D) data back to the user [13]. Figures 2(a), (b), 3 and $\mathbf{4}$ show typical images developed using laser, ultrasonic (UT) and acoustic (sonar) scans.

Figures 2(a) and (b) show typical images developed using a laser 3-D scanner system. This technology can also be applied to fibre optic systems. The laser or other optical waves could be used on sections of interest and imaged in a 3-D format.

Recently, ultrasonic output has significantly improved with the digital ultrasonic instruments. Depending on the inspection technology, ultrasonic testing machines have the capability to record ultrasonic data providing B-, C-, $\mathrm{D}$ - and P-Scans images represented in real time with hardcopy and file outputs. These are understandable to people with little or no knowledge about ultrasonic data $[14,15]$.

C-Scan obtained from ultrasonic (UT) represents the distribution of the informative parameter over the top surface of the area of interest in the form of a projection map. B-Scan represents the sectional views of the object under test. When the depth projection is considered, it is

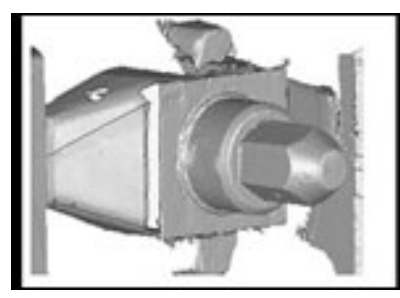

(a)

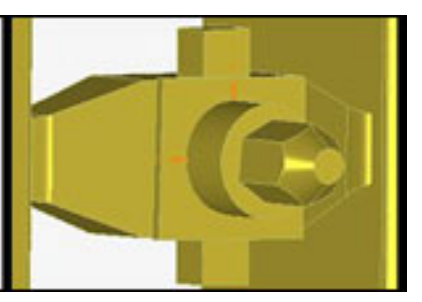

(b)
Figure 2. Use of industry standard (a) 3-D laser scanner and (b) CAD software on Laser Scanner (Source: [13]). referred to as a D-Scan. Combinations of B-, C- and DScans represent the P-Scan, yielding much detailed information. As with the laser system, when used with industry-standard 3-dimensional software, a full measurable computer-aided design model can be generated in some cases. Figure 3 shows a C-Scan of a composite spar presented in 3-dimensional imaging.

Acoustic imaging may be made by use of multi-beam and high resolution sonars [17]. A sample of acoustic (sonar) imaging capability of the acoustic scan can be seen in Figure 4.

The advantages and disadvantages of 3-dimensional imaging are summarized as follows:

Advantages:

1) They can be used for open, buried or submerged pipelines.

Disadvantages:

1) It is not possible to design a 3D imaging system that does not have some errors [19]. The recent proposal of a standard for assessing the performance of $3 \mathrm{D}$ imaging is a pointer that errors do exist in 3D imaging systems [20].

2) They are relatively expensive compared to close visual examination.

3) Expert equipment and operator are often required.

4) Expert interpretation may be required.

\section{Close Visual Inspection-Human Eye as Main Tool}

Close Visual Inspection is often made by use of human

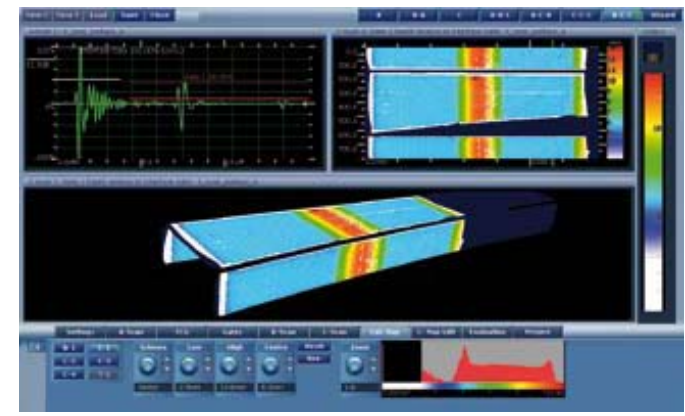

Figure 3. C-Scan map of a composite spar structure displayed on 3D image (Source: [16]).

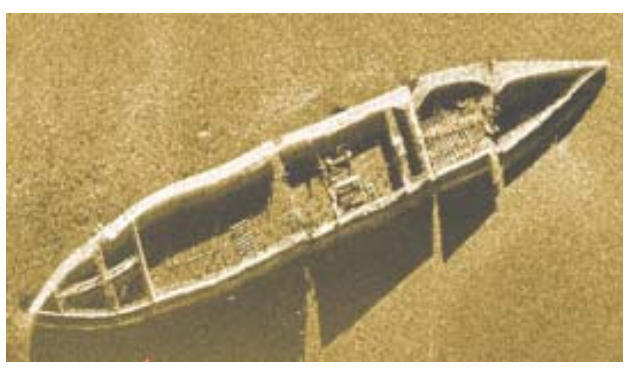

Figure 4. Showing a wreck at the sea bottom located by Acoustic (Sonar) Scan (Source: [18]). 
eye. It is the natural optical method of visualisation using the human eye as the main tool. It takes the form of close-look, close-up photography or close-up video of the subject matter. Recent work has shown that clear close-up photography and video can be made even in very muddy water by the use of laminar flow of clear water [21] or any other suitable technique. The system simply works by supplying laminar flow of clear water over the surface of the structure. A camera is then placed over the clear water to observe the structure and transmit the details to the surface engineer.

Where clarity and underwater visibility permits, it is obvious that an optical and visual observation using human eyes is relatively much better than any 3-D imaging in assessing and classifying damages adequately and timely. By being timely, it implies that for the JIV issues, controversies and disagreements between stakeholders are reduced for classification of pipeline breaks in unclear water condition.

Advantages:

1) It provides more convincing pictures than the use of 3-D imaging.

2) The image produced is more real than any 3-D imaging system.

3) Cheap to use.

4) No special expertise or training is required in its operation.

5) Environmentally friendly.

Disadvantage:

It is not used for buried or covered objects except where the seabed cover is removed.

Even with these benefits, there are still challenges. The laminar flow approach to optical visualisation in pipeline inspection in underwater poor visibility condition indicates that the tool would be hand-carried by divers. The divers would have to trace the position of the leaking point on the pipeline in unclear water mass. This is a challenge. The use of a remotely operated vehicle equipped with an acoustic system could provide an alternative to tracing the pipeline break point if the leak is from a pinhole under a high pressure pipeline system. However, considering old pipelines, where brittle fracture has taken place or initiated, pressurising the pipeline system to provide a track-able acoustic noise could result in brittle fracture propagation, therefore, serious pollution. It is therefore necessary to propose an extension of the laminar clear water technology to include a system requiring lower pressure in the pipeline system for leak detection.

\section{Perspective}

[21] demonstrates a method of pipeline observation in underwater poor visibility using laminar clear water flow- ing over the surface of the leaking pipeline as underwater camera is placed on top of the laminar water to observe the leaking structure. Similar system using displacement air instead of laminar clear water has been tested. These are within laboratory stages and it is necessary to further develop these techniques in the field.

The natural sequence of repairing an oil pipeline leak in most cases is to shut-down production and back-flush to minimize spills to the environment. Back-flushing is often achieved by batching clean water from the valve closest to the suspected leaking point, to displace the oil in the pipeline. This paper proposes an extension of this sequence to application of air/gas pressure in the pipeline at minimum pressure to reproduce gas plumes in order to identify locations of pipeline leaks from surface to subsurface of the water mass.

Publications [22] and [23] agree that an oil and gas plume that forms from leaks of oil and gas subsea pipeline are conical and rise vertically towards the surface with the plumes vertical momentum fading as the pressure drops or the water column gets larger. In otherwords, a plume can generate vertical velocity and acceleration of the motion of fluid. This can be achieved using relatively lower in-line pressure. The motion could be tracked and used as a guide to track the pipeline point of leak. The principle is: where the break is on the surface of the pipeline, that is where the plume is coming from. In a clear water condition, simple equipment with video capability could be a simple guide to identify the leaking surface of the pipeline. In poor underwater visibility condition, it is suggested to use vertical motion sensor to approach or follow the plume gradually from top to bottom: where the momentum is coming from is where the break exist on the pipeline. The tracking would preferably, be made by an ROV that would remotely operate the close-visual inspection apparatus as in [21].

It is possible to design the tool such that should the pipeline be buried under soil cover, the equipment would have the capability to approach the identified pipeline section, jet away the surrounding soils and perform the clear observations to enable an adequate repair strategy.

\section{Conclusions}

This paper has attempted to document an approach to fill a gap in the present technology for the inspection of damaged submerged pipelines in unclear water conditions. The Close-Visual Inspection in its original meaning of using the optical human eyes as the main tool of observation cannot be substituted with any better alternative system. Even a close-up photography will contain more virtual details than any 3-D imaging of any technology as at today. 
In bridging this gap and providing solution to incessant leak-repair-leak cycle due to improper inspection and repair work in underwater pipelines where poor visibility is common, it is important to explore opportunities in laminar flow approach or related technique, and in combination with other existing technology, to extend the innovation to use remotely operated system by using the exiting plume as the tracking medium.

\section{Acknowledgements}

One expresses gratitude to AS Norske SHELL for provision of fund for the research studies in this subject area (project ${ }^{\#}$ 92917-University of Stavanger, Norway). I also remain indebted to my supervisors, Professor Gudmestad Ove T., and Professor Torliev Bilstad of the University of Stavanger for their unwavering guidance, tutoring and supports. One is also thankful to Uncle Levi Okoronkwo for his assistance in the field work.

\section{REFERENCES}

[1] J. Agbakwuru and O. T. Gudmestad, "Integrity of Pipeline: Some Challenges and Perspectives," 2nd Annual Global Pipeline Integrity Interactive Technology Workshop, Istanbul, 28 November-1 December 2011, Praxis Global Research.

[2] E. Ugwuani, "Shell Records 2580 Barrel Oil Spill," The Nations, 2011.

http://www.thenationonlineng.net/2011/index.php/busines s/energy/8578-shell-records-2-580-barrels-oil-spill.html

[3] P. Hopkins, "The Structural Integrity of Oil and Gas Transmission Pipelines," Comprehensive Structural Integrity, Elsevier Publishers Penspen Ltd., Berlin, 2002.

[4] H. H. Sayyed, "Apportion of Charpy Energy in API 5L Grade X70 Pipeline Steel,” International Journal of Pressure Vessels and Piping, Vol. 85, No. 12, 2008, pp. 879884. doi:10.1016/j.ijpvp.2008.04.011

[5] P. Hopkins, "Defect Assessment in Pipelines,” Amsterdam Hilton Hotel, 28-29 October 2002, Penspen Group Limited.

[6] Onuoha, "Oil Pipeline Sabotage in Nigeria: Dimensions, Actors and Implications for National Security," African Security Review, Vol. 17, No. 3, 2008, pp. 99-115. doi:10.1080/10246029.2008.9627487

[7] J. O. Arumala and E. G. Akpokodje, "Soil Properties and Pavement Performance in the Niger Delta," Quarterly Journal of Engineering Geology and Hydrogeology, Vol. 20, No. 4, 1987, pp. 287-296. doi:10.1144/GSL.QJEG.1987.020.04.05

[8] D. N. Veritas, "Recommended Practice, DNV-RP-F113 Pipeline Subsea Repair,” Det Norske Veritas, Høvik, 2007, pp. 5-6.

[9] Amnesty International Report, "Nigeria: Petroleum, Pollution and Poverty in the Niger Delta," Amnesty International, 9 June 2009, Index: AFR 44/017/2009.

[10] J. Bevan, “The Professional Diver's Handbook,” 2nd Edition, Submex Limited Hampshire, Hampshire, 2009.

[11] T. K. Fanneløp, "Flow Processes and Leak Rates Associated with Broken Underwater Pipelines," Norwegian Maritime Research, Vol. 5, No. 1, 1977, pp. 6-13.

[12] T. Chris, "Subsea Leak Detection,” Neptune Oceanographics Ltd., Carlbury, 2007.

[13] Newton Labs, “Nuclear Laser Scanner,” Seattle, 2011. http://www.photonics.com/Article.aspx?AID=45956

[14] V. Moshkovich, A. Passi, G. Passi and R. Safin, "Recent Advances in the Ultrasonic Inspection Recording and Reporting-Instrumentation Part 2,” NDT Net, Vol. 8, No. 11, 2003 http://www.ndt.net/article/v08n11/passi2/passi2.htm

[15] V. Moshkovich, A. Passi and G. Passi, "Recent Advances in the Ultrasonic Inspection Recording and ReportingInstrumentation Part 1,” NDT Net, Vol. 9. No. 9, 2003. http://www.ndt.net/article/v08n09/passi/passi.htm

[16] Sonatest NDTS. http://www.ndtsolutions.com/products/multi/rapidscan3d/ brochure/pdf/brochure.pdf

[17] H. Assalih, Y. Petillot and J. Bell, “Acoustic Stereo Imaging (ASI) System,” Oceans, 2010, pp. 1-7.

[18] EdgeTech, Affiliate of ORE Offshore. http://www.edgetech.com/

[19] Capturing Concept in 3D, 2011. http://www.3ddigitalcorp.com/3d-laser-scanner.shtml

[20] D. K. MacKinnon, "Assessing the Performance of 3DImaging Systems," SPIE, The International Society for Optics and Photonics, 9 March 2011. http://spie.org/x44547.xml?ArticleID=x44547

[21] J. Agbakwuru, O. T. Gudmestad, J. C. Grønli and H. Skjaveland, "Development of Method/Apparatus for CloseVisual Inspection of Under-Water Structures (Especially Pipelines) in Muddy and Unclear Water Condition,” Proceedings of the $30^{\text {th }}$ International Conference on Ocean, Offshore and Arctic Engineering, Rotterdam, 19 June 2011, pp. 209-217.

[22] M. Reed, Ø. Johansen, B. HØverstad, B. Hetland, S. Buffington and M. H. Emilsen, "Numerical Model for Estimation of Pipeline Oil Spill Volumes, International Oil Spill Response: Oil Fate \& Transport Response,” Environmental Modelling \& Software, Vol. 21, No. 2, 2006, pp. 178-189.

[23] H. Rye and P. J. Brandvik, "Verification of Subsurface Oil Spill Models,” Proceedings of the 1997 International Oil Spill Conference, Fort Lauderdale, 7-10 April 1997, pp. 551-557. 